\title{
A New Grid-free Method for Conservation Laws
}

\author{
Praveen Chandrashekar and S M Deshpande \\ ARDB CFD Center, Department of Aerospace Engg., \\ Indian Institute of Science, Bangalore 560012, INDIA \\ Email: praveen@aero.iisc.ernet.in
}

\begin{abstract}
We present a grid-free or meshless approximation called the Kinetic Meshless Method (KMM), for the numerical solution of hyperbolic conservation laws that can be obtained by taking moments of a Boltzmann-type transport equation. The meshless formulation requires the domain discretization to have very little topological information; a distribution of points in the domain together with local connectivity information is sufficient. For each node $i$, the connectivity consists of a set of nearby nodes $\mathcal{C}_{i}$ which are used to evaluate the spatial derivatives appearing in the conservation law. The derivatives are obtained using a modified form of the least squares approximation. The method is applied to the Euler equations for inviscid flow and results are presented for some 2-D problems.
\end{abstract}

\section{Introduction}

Meshless methods are being increasingly proposed as an alternative to conventional grid-based methods for the solution of problems involving complex geometry and moving boundaries. They only require a distribution of points in the domain which can be obtained from either one or more of the available grid generation tools. For complex domains, the point distribution can also be obtained from overlapping chimera meshes and there is no need for interpolation at the boundaries of the overlap regions. Another option is to use quadtree/octree based grids which are relatively easier to generate and we do not have to worry about badly deformed or cut-cells near the boundaries. Thus a single (meshless) solver can be used on all types of grids or point distributions [1]. Recently some effort has been directed towards point generation techniques (Lohner \& Onate, Lee) also.

Meshless methods for compressible flows where the governing equations are hyperbolic have been developed by Ghosh and Deshpande [2,3], Batina [4], Morinishi [5], and Balakrishnan [6], all of which make use of a least squares formulation. They differ from one another in the way they introduce upwinding. They solve the governing equations in the pde form unlike many other meshless methods (Belytschko et. al., Junk et. al., Liu et. al.) which use a weak formulation and require the evaluation of many integrals over the computational domain. The LSKUM [3] of Ghosh and Deshpande is a kinetic meshless scheme and is obtained by taking moments of a meshless upwind discretization of the Boltzmann equation, and the same approach is taken in the present work. The present method differs from LSKUM in that it uses a different upwind-type discretization of the Boltzmann equation which requires less number of points 
in each connectivity. Kinetic schemes are attractive due to the fact that they are free of all tunable parameters and artificial fixes, and are known to be very robust in terms of positivity, entropy consistency and convergence history.

\section{Kinetic Representation}

We are interested in the numerical solution of hyperbolic conservation laws which can be obtained by taking suitable moments of a Boltzmann-type transport equation. The system of conservation laws is written in differential form as

$$
\frac{\partial U}{\partial t}+\sum_{\alpha=1}^{d} \frac{\partial F^{\alpha}}{\partial x^{\alpha}}=0
$$

where $U$ is the vector of conserved variables and $F^{\alpha}$ are the flux vectors. We assume that the system of equations (1) has a kinetic representation, that is, it can be obtained by taking moments of a Boltzmann equation

$$
\frac{\partial f}{\partial t}+\sum_{\alpha=1}^{d} v_{\alpha} \frac{\partial f}{\partial x^{\alpha}}=J(f)
$$

where the moments are defined as

$$
\langle\cdot\rangle=\int_{\mathbb{R}^{d} \times \mathbb{R}_{+}} \Psi(\vec{v}, I)(\cdot) \mathrm{d} \vec{v} \mathrm{~d} I
$$

Equation (1) is obtained by taking moments of (2) provided $\langle f\rangle=U,\left\langle v_{\alpha} f\right\rangle=$ $F^{\alpha}$ and $\langle J(f)\rangle=0$. The variable $I$ is sometimes necessary to get the correct moment closure and represents non-translational degrees of freedom. The kinetic representation gives a particle picture of the system under consideration, where the particles are moving with velocity $\vec{v}$, with the function $f(\vec{v}, I ; \vec{x}, t)$ giving the statistical distribution of molecular velocity. The macroscopic behaviour is obtained by taking average/moments of the microscopic behaviour. It has been shown by Harten, Lax and van Leer that (1) has a kinetic representation if either $F^{\alpha}(U)=A^{\alpha}(U) U$, with all the $A^{\alpha}$ having only real eigenvalues and a complete set of eigenvectors, or, if the system of equations (1) has a convex entropy. Conservation laws modeling a physical system will always be associated with an entropy condition due to the second law of thermodynamics. In such a case we can use the moment method strategy of Deshpande: first obtain an upwind discretization of (2) and take moments to obtain a discretization for the conservation law (1). Note that we do not actually solve equation (2) but merely use it in an intermediate step to obtain a discretization of (1).

\section{Dual Least Squares Approximation}

A variant of the least squares approximation, which we call the dual least squares approximation, has been used previously by some authors $[5,6]$. Let $f: \mathbb{R}^{d} \rightarrow \mathbb{R}$ 
be a sufficiently smooth function. If $j \in \mathcal{C}_{i}$ then let $f_{i j}$ denote the value of $f$ at the mid-point of the line segment $i \vec{j}$. Using Taylor's formula about the point $\vec{x}_{i}$, we obtain

$$
f_{i j}=f_{i}+\frac{1}{2} \Delta \vec{x}_{i j} \cdot \nabla f_{i}+O\left(\left|\Delta \vec{x}_{i j}\right|^{2}\right), \quad \Delta \vec{x}_{i j}=\vec{x}_{j}-\vec{x}_{i}
$$

The dual least squares approximation for $\nabla f_{i}$ is obtained from the following weighted minimization:

$$
\operatorname{minimize} \sum_{j \in \mathcal{C}_{i}} w_{i j}\left[f_{i j}-f_{i}-\frac{1}{2} \Delta \vec{x}_{i j} \cdot \nabla f_{i}\right]^{2}, \quad \text { w.r.t. } \nabla f_{i}
$$

This gives a linear system of $d \times d$ equations, $\mathbb{A}_{i} \nabla f_{i}=\mathrm{b}_{i}$, where $\mathbb{A}_{i}$ is a square matrix that depends only on the coordinates of node $i$ and its neighbours. In the present work the weight function is chosen as $w_{i j}=\left|\vec{x}_{i}-\vec{x}_{j}\right|^{-2}$. The matrix $\mathbb{A}$ is symmetric and positive definite as long as the connectivity is non-degenerate; hence a unique solution always exists. Moreover all the elements of $\mathbb{A}$ have the same dimensions which leads to good condition numbers. The solution of the above linear system can be written as

Dual Least Squares (DLS): $\left.\frac{\partial f}{\partial x^{\alpha}}\right|_{i}=\sum_{j \in \mathcal{C}_{i}} \mu_{i j}^{\alpha}\left(f_{i j}-f_{i}\right)$

In order to get first order accurate estimate of $\nabla f$, Balakrishnan [6] has shown that we must use atleast a second order estimate of $f_{i j}$, i.e., $f_{i j}=f\left(\left(\vec{x}_{i}+\vec{x}_{j}\right) / 2\right)+$ $O\left(\left|\vec{x}_{i}-\vec{x}_{j}\right|^{2}\right)$. We also mention the least squares approximation of the derivative,

Least Squares (LS): $\left.\frac{\partial f}{\partial x^{\alpha}}\right|_{i}=\frac{1}{2} \sum_{j \in \mathcal{C}_{i}} \mu_{i j}^{\alpha}\left(f_{j}-f_{i}\right)$

Equations (5), (6) show the difference between DLS and LS; in LS we make use of known nodal values of the function whereas in DLS we use the function values at the mid-points which are not known a priori and have to be reconstructed in some way. An important issue in DLS is to get suitable mid-point values for satisfying specified accuracy and upwinding.

\section{Kinetic Meshless Method using DLS}

The present method is built on the DLS approximation and the moment-method strategy. The advantage of DLS is that the value of $f_{i j}$ is not known a priori, and we can introduce upwinding into the scheme by defining $f_{i j}$ in a consistent and upwind manner. Substituting the DLS estimate given by equation (5) into the Boltzmann equation and assuming that the collision term is identically zero, we obtain

$$
\frac{\mathrm{d} f_{i}}{\mathrm{~d} t}+\sum_{\alpha} \sum_{j \in \mathcal{C}_{i}} \mu_{i j}^{\alpha}\left(v_{\alpha} f_{i j}-v_{\alpha} f_{i}\right)=0
$$


Taking moments we obtain a semi-discrete form of the conservation law at node $i$

$$
\frac{\mathrm{d} U_{i}}{\mathrm{~d} t}+\sum_{\alpha} \sum_{j \in \mathcal{C}_{i}} \mu_{i j}^{\alpha}\left(F_{i j}^{\alpha}-F_{i}^{\alpha}\right)=0
$$

In order to upwind-bias the approximation given by equation (8), we construct $f_{i j}$ from the particle distribution functions of nodes $i$ and $j$, depending on whether the particle velocity has a component towards node $i$ or towards node $j$. If $V$ is a complete set of macroscopic variables which uniquely define $f$, then this approximation is given by

$$
f_{i j}(\vec{v}, I)= \begin{cases}f\left(\vec{v}, I ; V_{i j}^{+}\right), & \vec{v} \cdot \hat{e}_{i j} \geq 0 \\ f\left(\vec{v}, I ; V_{i j}^{-}\right), & \vec{v} \cdot \hat{e}_{i j} \leq 0\end{cases}
$$

where $\hat{e}_{i j}$ is the unit vector which is directed from node $i$ to node $j$. The $V_{i j}^{ \pm}$ must be atleast second-order accurate interpolants $[6,7]$ of $V$ from $\vec{x}_{i}, \vec{x}_{j}$ to the mid-point of $\overrightarrow{i j}$. This is achieved by using linear interpolation

$$
V_{i j}^{+}=R_{i j}\left(V_{i}+\frac{1}{2} \Delta \vec{x}_{i j} \cdot \nabla V_{i}\right), \quad V_{i j}^{-}=R_{i j}\left(V_{j}-\frac{1}{2} \Delta \vec{x}_{i j} \cdot \nabla V_{j}\right)
$$

The matrix $R_{i j}$ effects a rotation of the coordinate system such that the $x^{1}$-axis becomes parallel to $\hat{e}_{i j}$ upon rotation. This transformation makes it easier to use equation (10) in (9). The derivatives of $V$ are calculated using the least squares approximation given by equation (6). We can show [7] that the scheme given by (8), (9), (10) is rotationally invariant, i.e., if $U^{n+1}, U^{n+1}$ are the updated values in two Cartesian coordinate frames, then they are related by $\bar{U}^{n+1}=R U^{n+1}$, where $R$ is the coordinate transformation operator. Numerical experiments on a scalar conservation law indicate an effective accuracy of two even on highly non-uniform grids. Higher order schemes can be easily derived by including more terms in the Taylor's formula but higher order limiting for discontinuous flows is still an unsolved problem, especially on non-structured grids. It must be mentioned that we use the word upwinding in a somewhat general sense (upwind biased) and not in the usual sense that the update at a node is influenced by the data upwind of the node. We obtain mid-point values based on sign of signal propagation and then use full stencil in DLS. This hopefully gives enough dissipation to obtain a stable scheme and the large amount of numerical experience corroborates this expectation.

\section{$5 \quad$ Numerical Results}

We have applied KMM to solve the 2-D Euler equations governing inviscid compressible flows. These can be obtained by taking moments of the Boltzmann equation together with the Maxwellian distribution function. For interpolation, the primitive variables $V=\left[\begin{array}{lll}\rho, & \vec{u}, & p\end{array}\right]^{\top}$ are used; in the case of discontinuous flows, the gradients of $V$ are limited using Venkatakrishnan limiter. The wall 
boundary conditions are based on the strong formulation [8]. The point discretization is obtained from standard triangular grid generation techniques and the average number of points in the connectivity is six. The coefficients $\mu$ in (5) are calculated once in the beginning and stored (average of 12 floating point numbers per node in 2D) which reduces the cost of each time integration step. The first case is flow over the two-element Williams airfoil at $M_{\infty}=0.15, \alpha=0^{\circ}$ and is computed on a discretization of 6415 points with 233 points on the main airfoil and 120 on the flap. Figure (1a)-(1b) show comparison of the pressure coefficient between present results and the exact incompressible potential solution. The minimum and maximum entropy in the domain are $S_{\min }=3.45724$, $S_{\max }=3.46488$ while the free-stream value is $S_{\infty}=3.45776$, the maximum change being $0.22 \%$ of the freestream value. The lift and drag coefficients are 3.7608 and -0.0069 while the potential solution gives 3.736 and -0.0001 respectively. The second test case is flow over cylinder at $M_{\infty}=0.38$ on a discretization of 4111 points with 250 points on the cylinder. Figure (2) shows the Mach number variation on the cylinder which is seen to have good left-right and topbottom symmetry. The entropy production is very less with $S_{\min }=1.59731$, $S_{\max }=1.59886$ while the free-stream value is $S_{\infty}=1.59869$, the maximum change being $0.097 \%$ of the freestream value. The lift and drag coefficients are 0.0006 and 0.0012 respectively.

The third case is transonic flow over NACA-0012 at $M_{\infty}=0.85, \alpha=1^{\circ}$ which is computed on a discretization of 4385 points and another computation is performed with five levels of solution based adaptation, with the final grid having 6938 points. Figure (3) shows the pressure coefficient on the un-adapted and adapted grids and figure (4) shows the pressure contours on the final level of adapted grid. Finally, supersonic flow over a cylinder at $M_{\infty}=3.0$ is computed with 12857 points. The interior scheme is applied on the wall boundary points also and no special treatment is done except to kill the normal component of the velocity. The Mach contours are shown in figure (5) which show the bow shock on the front and the slip line on the leeward side of the cylinder. At the stagnation point behind the shock, the computed total pressure and total temperature are 0.3234 and 1.0006 , while the exact values are 0.3283 and 1.0000 respectively.

\section{Summary}

A new meshless method for numerical solution of hyperbolic conservation laws derivable from kinetic equations is described and applied to 2-D inviscid compressible flows. The results show good resolution of the flow features over a range of Mach numbers and are comparable to second order methods, with a numerical order of accuracy of two for a scalar conservation law. Combined with the strong wall boundary condition, it produces little spurious entropy as shown in the subsonic cases.

\section{References}

1. Ramesh V and Deshpande SM, ICCFD-1, Kyoto, 2000. 
2. Mandal, Ghosh and Deshpande, FM Report 1989-04, Dept. of Aerospace Engg., IISc, Bangalore, 1989.

3. Ghosh AK and Deshpande SM, AIAA Paper 95-1735, 1995.

4. Batina JT, AIAA Paper 93-0333, 1993.

5. Morinishi K, Int. Symp. on CFD, Bremen, 1999.

6. Sridar D and Balakrishnan N, FM Report 2001-01, Dept. of Aerospace Engg., IISc, Bangalore, 2001.

7. Praveen C and Deshpande S M, FM Report 2001-08, Dept. of Aerospace Engg., IISc, Bangalore, 2001.

8. Balakrishnan N and Fernandez G, Int. Jl. Num. Meth. Fluids, Vol. 28, pp. 14811501, 1998.

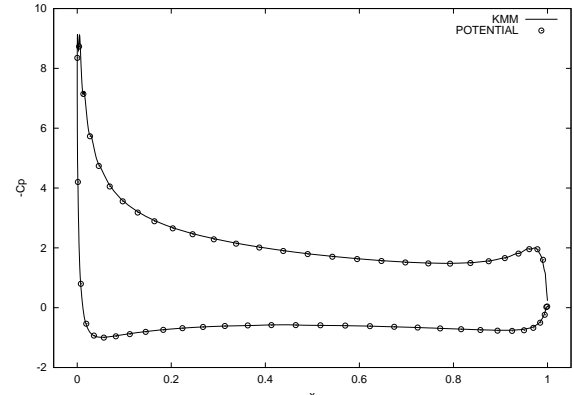

(1a)

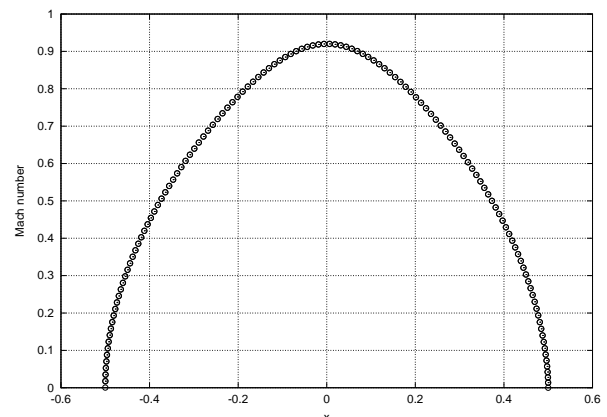

$(2)$

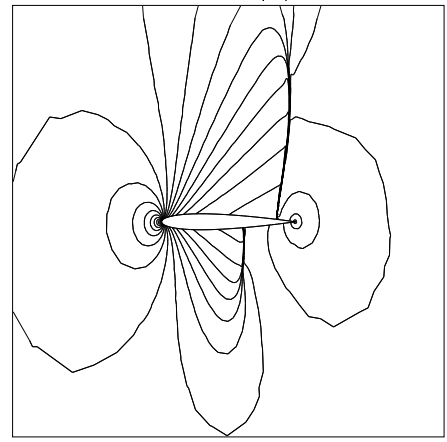

(4)

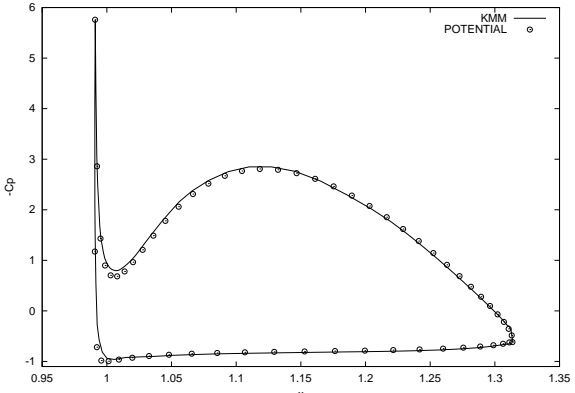

(1b)

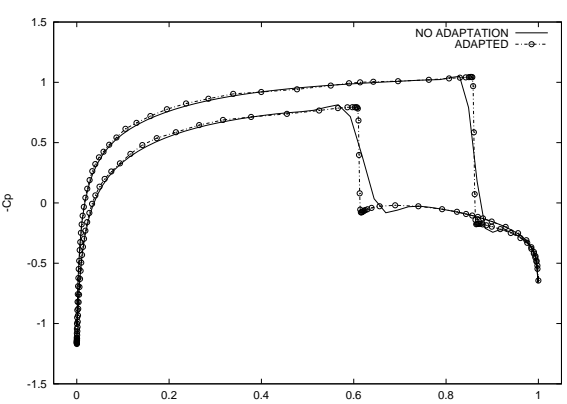

(3)

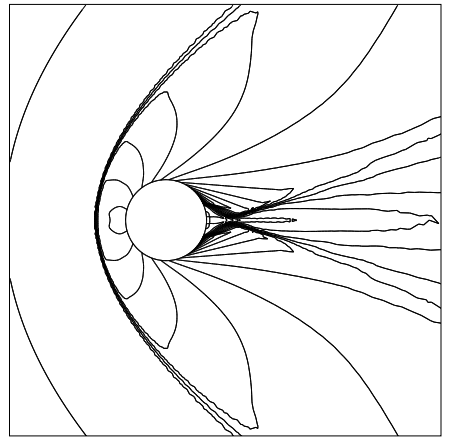

(5) 